\title{
PRESSURE JUMP CONDITIONS FOR STOKES EQUATIONS WITH DISCONTINUOUS VISCOSITY IN 2D AND 3D*
}

\author{
KUZUFUMI $\mathrm{ITO}^{\dagger}$, ZHILIN LI ${ }^{\ddagger}$, AND XIAOHAI WAN ${ }^{\S}$ \\ Dedicated to Björn Engquist's 60th Birthday
}

\begin{abstract}
In this paper, the jump conditions for the normal derivative of the pressure have been derived for two-phase Stokes (and Navier-Stokes) equations with discontinuous viscosity and singular sources in two and three dimensions. While different jump conditions for the pressure and the velocity can be found in the literature, the jump condition of the normal derivative of the pressure is new. The derivation is based on the idea of the immersed interface method $[9,8]$ that uses a fixed local coordinate system and the balance of forces along the interface that separates the two phases. The derivation process also provides a way to compute the jump conditions. The jump conditions for the pressure and the velocity are useful in developing accurate numerical methods for two-phase Stokes equations and Navier-Stokes equations.
\end{abstract}

Key words. incompressible Stokes equations, interface problem, discontinuous viscosity, singular sources, pressure jump condition

AMS subject classifications. 76D05

1. Introduction. The primary goal of this paper is to derive the jump condition of the normal derivative of the pressure for incompressible Stokes equations with discontinuous viscosity and singular sources along an interface in two and three dimensions. Since the inertial term $\rho \frac{D \boldsymbol{u}}{D t}$ in Navier-Stokes equations is continuous, the jump conditions of the velocity and the pressure for the Navier-Stokes equations are the same as the Stokes equations.

The governing equations with an immersed interface can be written as

$$
\begin{aligned}
\operatorname{div} \boldsymbol{\sigma}+\boldsymbol{G}+\boldsymbol{f}(\boldsymbol{x}) \delta_{\Gamma} & =0, \quad \boldsymbol{x} \in \Omega \subset R^{d}, \quad d=2,3, \\
\operatorname{div} \boldsymbol{u} & =0,
\end{aligned}
$$

where $\boldsymbol{G}$ is a body force, $\boldsymbol{f}$ is the density function of a surface force along the interface $\Gamma$ that separates the two phases, see Fig. 1 for an illustration. The stress tensor $\boldsymbol{\sigma}$ is given by

$$
\boldsymbol{\sigma}=-p \boldsymbol{I}+2 \mu \boldsymbol{T}
$$

and the strain rate tensor $\boldsymbol{T}$ is defined by

$$
T_{i j}=\frac{1}{2}\left(\frac{\partial u_{i}}{\partial x_{j}}+\frac{\partial u_{j}}{\partial x_{i}}\right),
$$

where $\boldsymbol{u}=u_{i}$ is the divergence free velocity field and $p$ is the pressure. The domain $\Omega$ is divided into two sub-domains $\Omega^{+}$and $\Omega^{-}$by $\Gamma \in C^{2}$, which we assume to be a smooth curve/surface within the solution domain.

\footnotetext{
* Received November 30, 2005; accepted for publication January 12, 2007.

${ }^{\dagger}$ Center For Research in Scientific Computation \& Department of Mathematics, North Carolina State University, Raleigh, NC 27695-8205, USA (kito@math.ncsu.edu).

${ }^{\ddagger}$ Center For Research in Scientific Computation \& Department of Mathematics, North Carolina State University, Raleigh, NC 27695-8205, USA (zhilin@math.ncsu.edu).

§Biomathematics Graduate Program, North Carolina State University, Raleigh, NC 27695-8203, USA (robertwan94@yahoo.com).
} 
We assume the viscosity coefficient $\mu$ is a piecewise constant,

$$
\mu(\boldsymbol{x})= \begin{cases}\mu^{+}, & \boldsymbol{x} \in \Omega^{+}, \\ \mu^{-}, & \boldsymbol{x} \in \Omega^{-} .\end{cases}
$$

For convenience of our discussion, we rewrite the incompressible Stokes equations as the following tensor form,

$$
\begin{gathered}
\frac{\partial p}{\partial x_{i}}=\frac{\partial}{\partial x_{j}}\left(\mu\left(\frac{\partial u_{i}}{\partial x_{j}}+\frac{\partial u_{j}}{\partial x_{i}}\right)\right)+G_{i}, \quad \boldsymbol{x} \in \Omega \backslash \Gamma, \\
\frac{\partial u_{i}}{\partial x_{i}}=0, \quad \boldsymbol{x} \in \Omega \backslash \Gamma,
\end{gathered}
$$

along with jump conditions across the interface $\Gamma$ and a boundary condition along $\partial \Omega$. The singular source term $\boldsymbol{f} \delta_{\Gamma}$ will be reflected in the jump conditions for the pressure and the velocity that couples the solutions in $\Omega^{+}$and $\Omega^{-}$regions.

Two-phase Stokes equations have many applications, see, for example $[3,6]$ and the reference therein. In order to get accurate numerical methods for solving two phase flows, it is helpful to know how the physical quantities such as the pressure and the velocity are coupled together across the interface. For continuous viscosity, such interface relations and the derivations can be found in $[3,5,6]$.

(a).

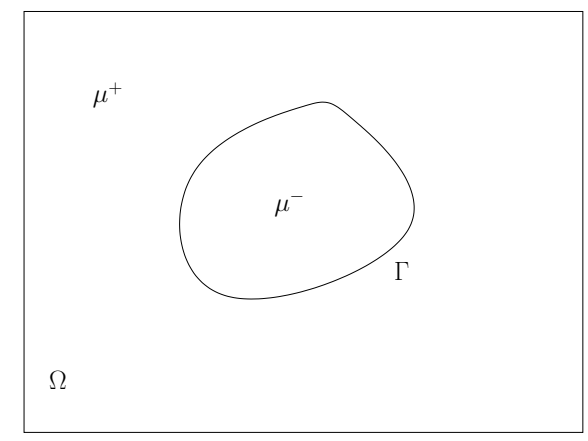

(b).

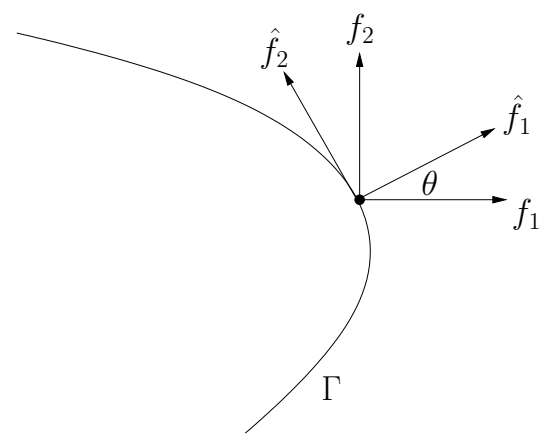

FIG. 1. (a). A diagram for $2 D$ incompressible Stokes equations defined on a domain $\Omega$ with an interface $\Gamma$ across which the viscosity $\mu$ is discontinuous. (b). The force density decomposition in which $f_{1}$ and $f_{2}$ are the force density components in the $x_{1}-$ and $x_{2}$-directions, while $\hat{f}_{1}$ and $\hat{f}_{2}$ are the force density components in the normal and tangential directions.

If the viscosity has a finite jump across the interface, while some of the interface relations are known in the literature, for example, [4, 2, 3], they may not be enough for some numerical methods. In [10], an immersed interface method that requires three fast Poisson solvers (in 2D) within each iteration was developed assuming that the jump in the viscosity is small so that the jump conditions can be approximated with those for a continuous viscosity, that is, the coupling terms are ignored. Such approximation may not be valid anymore if the jump in the viscosity is large. While [2] provided a new way of proving the jump conditions and some new results in two space dimensions, the result for the jump conditions of the normal derivative of the 
pressure missed an extra term which is corrected in this paper. In this paper, we use the idea of the immersed interface method to derive the jump condition for the normal derivative of the pressure in two and three dimensions. The idea is to use a fixed local Cartesian coordinate system built from the normal and tangential directions on the interface. The governing equations are invariant under such a coordinate transformation [12]. The interface and its geometrical information can be expressed easily in a neighborhood of the fixed point on the interface. The result presented here is new with mathematical formality. We also believe that this paper is the first to provide jump conditions for the pressure in component form. First we state the existing results of the jump conditions from the literature $[6,3]$.

THEOREM 1.1. Let $p$ and $\boldsymbol{u}=u_{i}$ be the solution to the Stokes equations (1.1), then the following jump conditions hold,

$$
\left[-p n_{i}+\mu\left(\frac{\partial u_{i}}{\partial x_{j}}+\frac{\partial u_{j}}{\partial x_{i}}\right) n_{j}\right]+f_{i}=0, \quad \text { or } \quad[p]=2\left[\mu \frac{\partial \boldsymbol{u}}{\partial n} \cdot \boldsymbol{n}\right]+\hat{f}_{1}
$$

in both two and three dimensions; and

$$
\left[\mu \frac{\partial u_{i}}{\partial n} \tau_{i}\right]+\left[\mu \frac{\partial u_{j}}{\partial \tau} n_{j}\right]+f_{i} \tau_{i}=0 \quad \text { or } \quad\left[\mu \frac{\partial \boldsymbol{u}}{\partial n} \cdot \boldsymbol{\tau}\right]+\left[\mu \frac{\partial \boldsymbol{u}}{\partial \tau} \cdot \boldsymbol{n}\right]+\hat{f}_{2}=0
$$

in two space dimensions. Since there are two orthogonal tangent directions in three dimension, there is an additional jump condition in the second tangent direction

$$
\left[\mu \frac{\partial u_{i}}{\partial n} \eta_{i}\right]+\left[\mu \frac{\partial u_{j}}{\partial \eta} n_{j}\right]+f_{i} \eta_{i}=0 \quad \text { or } \quad\left[\mu \frac{\partial \boldsymbol{u}}{\partial n} \cdot \boldsymbol{\eta}\right]+\left[\mu \frac{\partial \boldsymbol{u}}{\partial \eta} \cdot \boldsymbol{n}\right]+\hat{f}_{3}=0,
$$

in three dimensions. In the jump conditions above, $\boldsymbol{\tau}$ (or $\boldsymbol{\eta}$ ) is the unit tangential direction, $\boldsymbol{n}$ is the unit normal direction, $\hat{f}_{1}, \hat{f}_{2}$ and $\hat{f}_{3}$ are the force components in the normal and tangential directions, respectively.

The above theorem is the result of the balancing force in the normal and tangential directions on the interface.

Note that although the jump conditions are derived for Stokes equations in this paper, the results should be valid for Navier-Stokes equations with constant density since the material derivative is continuous. The results may be different for NavierStokes equations with variable density.

2. Local Cartesian coordinate systems. Our results and derivations are based on local coordinate systems built from the normal and tangential directions on the interface $\Gamma$. Let $\boldsymbol{X}^{*}=X_{j}^{*}$ be a point on the interface $\Gamma$, a local Cartesian coordinate system is one that centered at $\boldsymbol{X}^{*}$ with the axes being in the normal and tangential direction(s). Let the orthogonal transformation matrix be $A\left(A^{T} A=A A^{T}=I\right)$, we can write a corresponding Cartesian coordinate transformation as

$$
\tilde{x}_{i}=\left(A^{-1}\right)_{j}^{i}\left(x_{j}-X_{j}^{*}\right)
$$

In two space dimensions, for example, a local coordinate system can be written as

$$
\left\{\begin{array}{l}
\tilde{x}_{1}=\left(x_{1}-X_{1}^{*}\right) \cos \theta+\left(x_{2}-X_{2}^{*}\right) \sin \theta \\
\tilde{x}_{2}=-\left(x_{1}-X_{1}^{*}\right) \sin \theta+\left(x_{2}-X_{2}^{*}\right) \cos \theta
\end{array}\right.
$$


where $\theta$ is the angle between $x_{1}$ axis and the normal direction of the interface, see Fig. 1 (b) for an illustration. Note that the Stokes equations are invariant under an orthogonal coordinates transformation.

We will use $\tilde{u}_{1}$, for example, to represent the first component of the vector $\boldsymbol{u}$ in the local coordinate system. We will still use $p$ in the local coordinate system for simplicity since it is a scalar, that is, we use $p\left(\tilde{x}_{1}, \tilde{x}_{2}\right)=\tilde{p}\left(\tilde{x}_{1}, \tilde{x}_{2}\right)=p\left(x_{1}, x_{2}\right)$. This will not cause any confusion.

Under the local coordinates, the normal derivative of a two dimensional function, for example, $\tilde{u}_{1}\left(\tilde{x}_{1}, \tilde{x}_{2}\right)$, is defined as

$$
\frac{\partial \tilde{u}_{1}}{\partial \tilde{n}}=\left(\nabla_{\left(\tilde{x}_{1}, \tilde{x}_{2}\right)} \tilde{u}_{1}\right) \cdot \tilde{\boldsymbol{n}}
$$

where $\tilde{\boldsymbol{n}}$ is the unit normal vector in the local coordinates. Similarly, we define

$$
\frac{\partial \tilde{u}_{1}}{\partial \tilde{\tau}}=\left(\nabla_{\left(\tilde{x}_{1}, \tilde{x}_{2}\right)} \tilde{u}_{1}\right) \cdot \tilde{\boldsymbol{\tau}},
$$

as the tangential derivative of $\tilde{u}_{1}$, where $\tilde{\boldsymbol{\tau}}$ is the unit tangential vector in the local coordinates. The second order derivatives can be defined accordingly, for example,

$$
\frac{\partial^{2} \tilde{u}_{1}}{\partial \tilde{n} \partial \tilde{\tau}}=\frac{\partial}{\partial \tilde{n}}\left(\frac{\partial \tilde{u}_{1}}{\partial \tilde{\tau}}\right)
$$

We first prove several Lemmas that are going to be used in deriving the jump conditions.

LEMma 2.1. With the coordinates transformation (2.1), we have the following equality,

$$
\frac{\partial u_{i}}{\partial n} n_{i}=\frac{\partial \tilde{u}_{i}}{\partial \tilde{n}} \tilde{n}_{i}
$$

Proof.

$$
\begin{aligned}
\frac{\partial \tilde{u}_{i}}{\partial \tilde{n}} \tilde{n}_{i} & =\frac{\partial \tilde{u}_{i}}{\partial \tilde{x}_{k}} \tilde{n}_{k} \tilde{n}_{i} \\
& =\left(A^{-1}\right)_{j}^{i} \frac{\partial u_{j}}{\partial \tilde{x}_{k}} \tilde{n}_{k} \tilde{n}_{i} \quad\left(\text { since } \tilde{u}_{i}=\left(A^{-1}\right)_{j}^{i} u_{j}\right) \\
& =\left(A^{-1}\right)_{j}^{i}(A)_{k}^{l} \frac{\partial u_{j}}{\partial x_{l}}\left(A^{-1}\right)_{p}^{k} n_{p}\left(A^{-1}\right)_{q}^{i} n_{q} \\
& =\frac{\partial u_{i}}{\partial n} n_{i} .
\end{aligned}
$$

LEMma 2.2. With the coordinates transformation (2.1), the following equality is true,

$$
\frac{\partial^{2} u_{i}}{\partial x_{j} \partial x_{j}} n_{i}=\frac{\partial^{2} \tilde{u}_{i}}{\partial \tilde{x}_{j} \partial \tilde{x}_{j}} \tilde{n}_{i}
$$


Proof.

$$
\begin{aligned}
\frac{\partial^{2} \tilde{u}_{i}}{\partial \tilde{x}_{j} \partial \tilde{x}_{j}} \tilde{n}_{i} & =\left(A^{-1}\right)_{k}^{i} \frac{\partial^{2} u_{k}}{\partial \tilde{x}_{j} \partial \tilde{x}_{j}} \tilde{n}_{i} \\
& =\left(A^{-1}\right)_{k}^{i} \frac{\partial}{\partial \tilde{x}_{j}}\left((A)_{j}^{l} \frac{\partial u_{k}}{\partial x_{l}}\right) \tilde{n}_{i} \\
& =\left(A^{-1}\right)_{k}^{i}(A)_{j}^{l}(A)_{j}^{p}\left(A^{-1}\right)_{q}^{i} \frac{\partial^{2} u_{k}}{\partial x_{l} \partial x_{p}} n_{q} \\
& =\frac{\partial^{2} u_{i}}{\partial x_{j} \partial x_{j}} n_{i} .
\end{aligned}
$$

Lemma 2.3. With the coordinates transformation (2.1), the incompressibility is invariant, that is,

$$
\frac{\partial u_{i}}{\partial x_{i}}=\frac{\partial \tilde{u}_{i}}{\partial \tilde{x}_{i}}=0
$$

Proof.

$$
\begin{aligned}
\frac{\partial \tilde{u}_{i}}{\partial \tilde{x}_{i}} & =\left(A^{-1}\right)_{k}^{i} \frac{\partial u_{k}}{\partial \tilde{x}_{i}} \\
& =\left(A^{-1}\right)_{k}^{i}(A)_{i}^{l} \frac{\partial u_{k}}{\partial x_{l}} \\
& =\frac{\partial u_{i}}{\partial x_{i}}
\end{aligned}
$$

REMARK 2.4. Lemma 2.1-2.3 state that the governing equations are invariant under the local coordinates transformation.

2.1. A direct jump condition for $\frac{\partial p}{\partial n}$. From the momentum equation (1.5), we multiply $\boldsymbol{n}$ to both sides of the equation to get

$$
\begin{aligned}
\frac{\partial p}{\partial n} & =\frac{\partial}{\partial x_{j}}\left(\mu\left(\frac{\partial u_{i}}{\partial x_{j}}+\frac{\partial u_{j}}{\partial x_{i}}\right)\right) n_{i}+G_{i} n_{i} \quad x \in \Omega \backslash \Gamma \\
& =\mu\left(\frac{\partial^{2} u_{i}}{\partial x_{j} \partial x_{j}}+\frac{\partial^{2} u_{j}}{\partial x_{j} \partial x_{i}}\right) n_{i}+G_{i} n_{i} \quad \text { (since } \mu \text { is piecewise constant) } \\
& =\mu \frac{\partial^{2} u_{i}}{\partial x_{j} \partial x_{j}} n_{i}+G_{i} n_{i}, \quad \text { (from the incompressibility condition) } .
\end{aligned}
$$

Thus we have

$$
\left[\frac{\partial p}{\partial n}\right]=\left[\mu \frac{\partial^{2} u_{i}}{\partial x_{j} \partial x_{j}} n_{i}\right]+\left[G_{i} n_{i}\right]=[\mu \Delta \boldsymbol{u} \cdot \boldsymbol{n}]+[\boldsymbol{G} \cdot \boldsymbol{n}] .
$$

This is an obvious jump condition for $\frac{\partial p}{\partial n}$ but may not have practical use in developing numerical methods since it involves second order partial derivatives of the velocity. 
3. The derivation of the jump condition for $\frac{\partial p}{\partial n}$ in $2 \mathrm{D}$. In two space dimensions, the transformation matrix at a fixed point $\boldsymbol{X}^{*}$ on the interface is

$$
A=\left(\begin{array}{cc}
n_{1}\left(\boldsymbol{X}^{*}\right) & \tau_{1}\left(\boldsymbol{X}^{*}\right) \\
n_{2}\left(\boldsymbol{X}^{*}\right) & \tau_{2}\left(\boldsymbol{X}^{*}\right)
\end{array}\right)
$$

In a neighborhood of the origin of the local coordinate system, the interface can be represented by

$$
\Gamma=\left\{\left(\tilde{x}_{1}, \tilde{x}_{2}\right)=\left(\chi\left(\tilde{x}_{2}\right), \tilde{x}_{2}\right)\right\}
$$

where $\chi^{\prime}(0)=0$ and $\chi^{\prime \prime}(0)=\kappa$, here $\kappa$ is the curvature of the interface at the origin. We assume that the curvature of the unit circle is -1 . Therefore the local unit tangential direction is

$$
\tilde{\tau}=\frac{1}{\sqrt{1+\left(\chi^{\prime}\right)^{2}}}\left(\chi^{\prime}, 1\right)
$$

and the local unit normal direction is

$$
\tilde{\boldsymbol{n}}=\frac{1}{\sqrt{1+\left(\chi^{\prime}\right)^{2}}}\left(1,-\chi^{\prime}\right) .
$$

Using the idea of the level set function, we define $\varphi\left(\tilde{x}_{1}, \tilde{x}_{2}\right)=\tilde{x}_{1}-\chi\left(\tilde{x}_{2}\right)$ in a neighborhood of the origin of the local coordinate system. The zero level set of $\varphi\left(\tilde{x}_{1}, \tilde{x}_{2}\right)$ is the interface $\Gamma$. In a neighborhood of the origin, the normal direction is defined as $\tilde{\boldsymbol{n}}=\nabla \varphi\left(\tilde{x}_{1}, \tilde{x}_{2}\right) /\left|\nabla \varphi\left(\tilde{x}_{1}, \tilde{x}_{2}\right)\right|$, which is the same as that in (3.4). The tangential direction is also uniquely defined according to (3.3) in the same neighborhood and will be denoted as $\tilde{\boldsymbol{\tau}}$. In other words, with the level set function, we extend the normal and tangential directions to a neighborhood of the interface. We refer the readers to [11] for a reference about more general extensions of quantities off the curve and the discussion of the first and second order surface derivatives. true,

Lemma 3.1. For a given function $w\left(x_{1}, x_{2}\right)=w\left(\tilde{x}_{1}, \tilde{x}_{2}\right)$, the following equality is

$$
\frac{d[w]}{d \tilde{x}_{2}}(0)=\left[\frac{\partial w}{\partial \tilde{x}_{2}}\right](0)
$$

Proof. The left hand side of (3.5) is

$$
\begin{aligned}
\frac{d w^{+}}{d \tilde{x}_{2}}(0)-\frac{d w^{-}}{d \tilde{x}_{2}}(0) & =\frac{\partial w^{+}}{\partial \tilde{x}_{1}}(0) \frac{d \chi}{d \tilde{x}_{2}}(0)+\frac{\partial w^{+}}{\partial \tilde{x}_{2}}(0)-\left(\frac{\partial w^{-}}{\partial \tilde{x}_{1}}(0) \frac{d \chi}{d \tilde{x}_{2}}(0)+\frac{\partial w^{-}}{\partial \tilde{x}_{2}}(0)\right) \\
& =\frac{\partial w^{+}}{\partial \tilde{x}_{2}}(0)-\frac{\partial w^{-}}{\partial \tilde{x}_{2}}(0) \\
& =\left[\frac{\partial w}{\partial \tilde{x}_{2}}\right](0) .
\end{aligned}
$$

This completes the proof of the lemma.

REMARK 3.2. Since $[w]=[w]\left(\tilde{x}_{1}=\chi\left(\tilde{x}_{2}\right), \tilde{x}_{2}\right), \frac{d[w]}{d \tilde{x}_{2}}$ in (3.5) is calculated using the chain rule. 
One of our new results of this paper is the following theorem.

TheOREm 3.3. Let $\boldsymbol{X}^{*}$ be a point on the interface $\Gamma \in C^{2}$. Let $\left\{\tilde{x}_{j}\right\}=$ $\left(\boldsymbol{n}\left(\boldsymbol{X}^{*}\right), \boldsymbol{\tau}\left(\boldsymbol{X}^{*}\right)\right), j=1,2$, be the local coordinate system determined by (2.1). Then the following is true

$$
\left[\frac{\partial p}{\partial \tilde{n}}\right]=[\tilde{\boldsymbol{G}} \cdot \tilde{\boldsymbol{n}}]+\frac{d \hat{f}_{2}}{d \tilde{x}_{2}}+2\left[\mu \frac{\partial^{2} \tilde{u}_{1}}{\partial \tilde{x}_{2} \partial \tilde{x}_{2}}\right]-4 \kappa\left[\mu \frac{\partial \tilde{u}_{2}}{\partial \tilde{x}_{2}}\right]
$$

at the origin $\left(\tilde{x}_{j}=0\right)$ in the new coordinate system, where $\kappa$ is the curvature of the interface $\Gamma$ at $\boldsymbol{X}^{*}$, and $\tilde{u}_{1}=\boldsymbol{u} \cdot \boldsymbol{n}\left(\boldsymbol{X}^{*}\right)$ and $\tilde{u}_{2}=\boldsymbol{u} \cdot \boldsymbol{\tau}\left(\boldsymbol{X}^{*}\right)$ are the velocity component in the normal and tangential direction. Note that all the quantities in the equality above are evaluated at the origin of the new coordinates with the fixed local coordinate system.

Note that the following equalities hold,

$$
\left[\frac{\partial p}{\partial \tilde{n}}\right]=\left[\frac{\partial p}{\partial n}\right], \quad[\tilde{\boldsymbol{G}} \cdot \tilde{\boldsymbol{n}}]=[\boldsymbol{G} \cdot \boldsymbol{n}]
$$

Proof. Using Lemma 2.2, and from the representation of (2.13) (note that the Stokes equations are invariant in the orthogonal coordinate system), we have

$$
\left[\frac{\partial p}{\partial \tilde{n}}\right](0)=\left[\mu\left(\frac{\partial^{2} \tilde{u}_{1}}{\partial \tilde{x}_{1} \partial \tilde{x}_{1}}+\frac{\partial^{2} \tilde{u}_{1}}{\partial \tilde{x}_{2} \partial \tilde{x}_{2}}\right)\right](0)+\left[\tilde{G}_{i} \tilde{n}_{i}\right](0) .
$$

Rewriting equation (1.8) in $\tilde{x}_{i}$ coordinates, we get

$$
\left[\mu\left(\frac{\partial \tilde{u}_{i}}{\partial \tilde{n}} \tilde{\tau}_{i}+\frac{\partial \tilde{u}_{j}}{\partial \tilde{\tau}} \tilde{n}_{j}\right)\right]+\tilde{f}_{i} \tilde{\tau}_{i}=0 .
$$

We differentiate the equation (3.10) with respect to $\tilde{x}_{2}$ and evaluate at $\tilde{x}_{2}=0$ to get [12]

$$
\left[\mu \frac{\partial}{\partial \tilde{x}_{2}}\left(\frac{\partial \tilde{u}_{i}}{\partial \tilde{n}} \tilde{\tau}_{i}+\frac{\partial \tilde{u}_{j}}{\partial \tilde{\tau}} \tilde{n}_{j}\right)\right](0)+\frac{d\left(\tilde{f}_{i} \tilde{\tau}_{i}\right)}{d \tilde{x}_{2}}(0)=0 .
$$

The crucial step of the proof is to simplify the expression above at the origin of the new coordinate system, and combine (3.9) to get the desired result.

We expand the content of the first term inside [ · ] in expression (3.11) and evaluate its value at the origin to get (after some manipulations [12])

$$
\begin{aligned}
\left(\mu \frac{\partial}{\partial \tilde{x}_{2}}\left(\frac{\partial \tilde{u}_{i}}{\partial \tilde{n}} \tilde{\tau}_{i}+\frac{\partial \tilde{u}_{j}}{\partial \tilde{\tau}} \tilde{n}_{j}\right)\right)^{+}(0) & =\left(\mu\left(\frac{\partial^{2} \tilde{u}_{i}}{\partial \tilde{x}_{2} \partial \tilde{n}} \tilde{\tau}_{i}+\frac{\partial \tilde{u}_{i}}{\partial \tilde{n}} \frac{\partial \tilde{\tau}_{i}}{\partial \tilde{x}_{2}}+\frac{\partial^{2} \tilde{u}_{j}}{\partial \tilde{x}_{2} \partial \tilde{\tau}} \tilde{n}_{j}+\frac{\partial \tilde{u}_{j}}{\partial \tilde{\tau}} \frac{\partial \tilde{n}_{j}}{\partial \tilde{x}_{2}}\right)\right)^{+}(0) \\
& =\left(\mu\left(-2 \kappa \frac{\partial \tilde{u}_{2}}{\partial \tilde{x}_{2}}+\frac{\partial^{2} \tilde{u}_{2}}{\partial \tilde{x}_{1} \partial \tilde{x}_{2}}+2 \kappa \frac{\partial \tilde{u}_{1}}{\partial \tilde{x}_{1}}+\frac{\partial^{2} \tilde{u}_{1}}{\partial \tilde{x}_{2} \partial \tilde{x}_{2}}\right)\right)^{+}(0) .
\end{aligned}
$$

Note that the above simplifications utilize the definition of the directional derivatives, the fact that $\chi(0)=\chi^{\prime}(0)=0, \chi^{\prime \prime}(0)=\kappa$, and formulas like [12]

$$
\begin{aligned}
\frac{\partial^{2} \tilde{u}_{i}}{\partial \tilde{x}_{2} \partial \tilde{n}}= & \frac{\partial}{\partial \tilde{x}_{2}}\left\{\frac{1}{\sqrt{1+\left(\chi^{\prime}\right)^{2}}}\left(\frac{\partial \tilde{u}_{i}}{\partial \tilde{x}_{1}}-\frac{\partial \tilde{u}_{i}}{\partial \tilde{x}_{2}} \chi^{\prime}\right)\right\}=\frac{-\chi^{\prime} \chi^{\prime \prime}}{\left(1+\left(\chi^{\prime}\right)^{2}\right)^{2}}\left(\frac{\partial \tilde{u}_{i}}{\partial \tilde{x}_{1}}-\frac{\partial \tilde{u}_{i}}{\partial \tilde{x}_{2}} \chi^{\prime}\right) \\
& +\frac{1}{1+\left(\chi^{\prime}\right)^{2}}\left(\frac{\partial^{2} \tilde{u}_{i}}{\partial \tilde{x}_{1} \partial \tilde{x}_{1}} \chi^{\prime}-\frac{\partial^{2} \tilde{u}_{i}}{\partial \tilde{x}_{1} \partial \tilde{x}_{2}}\left(\chi^{\prime}\right)^{2}+\frac{\partial^{2} \tilde{u}_{i}}{\partial \tilde{x}_{1} \partial \tilde{x}_{2}}-\frac{\partial^{2} \tilde{u}_{i}}{\partial \tilde{x}_{2} \partial \tilde{x}_{2}} \chi^{\prime}-\frac{\partial \tilde{u}_{i}}{\partial \tilde{x}_{2}} \chi^{\prime \prime}\right) .
\end{aligned}
$$


Other terms are treated in the similar manner. We add (3.9) and (3.11) together to get

$$
\begin{aligned}
{\left[\frac{\partial p}{\partial \tilde{n}}\right](0)=} & \frac{d\left(\tilde{f}_{i} \tilde{\tau}_{i}\right)}{d \tilde{x}_{2}}(0)+\left[\tilde{G}_{i} \tilde{n}_{i}\right](0)+\left[\mu\left(\frac{\partial^{2} \tilde{u}_{1}}{\partial \tilde{x}_{1} \partial \tilde{x}_{1}}+\frac{\partial^{2} \tilde{u}_{1}}{\partial \tilde{x}_{2} \partial \tilde{x}_{2}}\right)\right](0) \\
& +\left[\mu\left(-2 \kappa \frac{\partial \tilde{u}_{2}}{\partial \tilde{x}_{2}}+\frac{\partial^{2} \tilde{u}_{2}}{\partial \tilde{x}_{1} \partial \tilde{x}_{2}}+2 \kappa \frac{\partial \tilde{u}_{1}}{\partial \tilde{x}_{1}}+\frac{\partial^{2} \tilde{u}_{1}}{\partial \tilde{x}_{2} \partial \tilde{x}_{2}}\right)\right](0) .
\end{aligned}
$$

From the incompressibility condition (Lemma 2.3) we have

$$
\frac{\partial^{2} \tilde{u}_{1}}{\partial \tilde{x}_{1} \partial \tilde{x}_{1}}+\frac{\partial^{2} \tilde{u}_{2}}{\partial \tilde{x}_{1} \partial \tilde{x}_{2}}=0 \quad \text { or } \quad \frac{\partial^{2} \tilde{u}_{1}}{\partial \tilde{x}_{1} \partial \tilde{x}_{1}}=-\frac{\partial^{2} \tilde{u}_{2}}{\partial \tilde{x}_{1} \partial \tilde{x}_{2}}
$$

Plugging this into (3.12) and making use of $\frac{\partial \tilde{u}_{1}}{\partial \tilde{x}_{1}}=-\frac{\partial \tilde{u}_{2}}{\partial \tilde{x}_{2}}$, we get

$$
\begin{aligned}
{\left[\frac{\partial p}{\partial \tilde{n}}\right](0) } & =\left\{\frac{d\left(\tilde{f}_{i} \tilde{\tau}_{i}\right)}{d \tilde{x}_{2}}+2\left[\mu \frac{\partial^{2} \tilde{u}_{1}}{\partial \tilde{x}_{2} \partial \tilde{x}_{2}}\right]-2 \kappa\left[\mu \frac{\partial \tilde{u}_{2}}{\partial \tilde{x}_{2}}\right]+2 \kappa\left[\mu \frac{\partial \tilde{u}_{1}}{\partial \tilde{x}_{1}}\right]+\left[\tilde{G}_{i} \tilde{n}_{i}\right]\right\}(0) \\
& =\left\{\frac{d\left(\tilde{f}_{i} \tilde{\tau}_{i}\right)}{d \tilde{x}_{2}}+2\left[\mu \frac{\partial^{2} \tilde{u}_{1}}{\partial \tilde{x}_{2} \partial \tilde{x}_{2}}\right]-4 \kappa\left[\mu \frac{\partial \tilde{u}_{2}}{\partial \tilde{x}_{2}}\right]+\left[\tilde{G}_{i} \tilde{n}_{i}\right]\right\}(0),
\end{aligned}
$$

after some simplification [12]. The expression above is the same as (3.7). This completes the proof.

\section{REMARK 3.4.}

- The jump condition of the normal derivative of the pressure can be written as

$$
\left[\frac{\partial p}{\partial n}\right]=[\boldsymbol{G} \cdot \boldsymbol{n}]+\frac{d}{d \tilde{x}_{2}}(\boldsymbol{f} \cdot \boldsymbol{\tau})+2\left[\mu \frac{\partial^{2} \boldsymbol{u}}{\partial \tilde{x}_{2} \partial \tilde{x}_{2}}\right] \cdot \boldsymbol{n}-4 \kappa\left[\mu \frac{\partial \boldsymbol{u}}{\partial \tilde{x}_{2}}\right] \cdot \boldsymbol{\tau},
$$

with the understanding that the all the quantities are evaluated at the fixed point.

- If the viscosity is continuous, we recover the jump condition for the normal derivative of the pressure derived in [6, 7] which is simply [12]

$$
\left[\frac{\partial p}{\partial n}\right]=[\boldsymbol{G} \cdot \boldsymbol{n}]+\frac{d}{d \tilde{x}^{2}}(\boldsymbol{f} \cdot \boldsymbol{\tau})
$$

3.1. An example in $\mathbf{2 D}$. We present an example here to verify the jump condition for $\left[p_{n}\right]$. We consider the following exact solution: 


$$
\begin{aligned}
& u_{1}= \begin{cases}\frac{x_{2}}{4}\left(\left(x_{1}\right)^{2}+\left(x_{2}\right)^{2}\right), & \boldsymbol{x} \in \Omega^{+}, \\
\frac{x_{2}}{4}, & \boldsymbol{x} \in \Omega^{-} .\end{cases} \\
& u_{2}= \begin{cases}-\frac{x_{1}\left(x_{2}\right)^{2}}{4}, & \boldsymbol{x} \in \Omega^{+}, \\
-\frac{x_{1}}{4}\left(1-\left(x_{1}\right)^{2}\right), & \boldsymbol{x} \in \Omega^{-} .\end{cases} \\
& p= \begin{cases}0, & \boldsymbol{x} \in \Omega^{+}, \\
\left(-\frac{3}{4}\left(x_{1}\right)^{3}+\frac{3}{8} x_{1}\right) x_{2}, & \boldsymbol{x} \in \Omega^{-} .\end{cases} \\
& g_{1}= \begin{cases}-2 x_{2}, & \boldsymbol{x} \in \Omega^{+}, \\
\left(-\frac{9}{4}\left(x_{1}\right)^{2}+\frac{3}{8}\right) x_{2}, & \boldsymbol{x} \in \Omega^{-} .\end{cases} \\
& g_{2}= \begin{cases}\frac{x_{1}}{2}, & \boldsymbol{x} \in \Omega^{+}, \\
-\frac{3}{4}\left(x_{1}\right)^{3}-\frac{3}{8} x_{1}, & \boldsymbol{x} \in \Omega^{-} .\end{cases} \\
& \mu= \begin{cases}1, & x \in \Omega^{+}, \\
\frac{1}{2}, & x \in \Omega^{-} .\end{cases}
\end{aligned}
$$

The interface is chosen to be the unit circle which is the zero level set of $\varphi\left(x_{1}, x_{2}\right)$,

$$
\varphi\left(x_{1}, x_{2}\right)=\sqrt{\left(x_{1}\right)^{2}+\left(x_{2}\right)^{2}}-1=0 .
$$

In the local Cartesian coordinates $\left(\tilde{x}_{1}, \tilde{x}_{2}\right)$, the interface is parameterized as

$$
\Gamma=\left(\tilde{x}_{1}, \tilde{x}_{2}\right)=\left(\chi\left(\tilde{x}_{2}\right), \tilde{x}_{2}\right)
$$

where $\chi\left(\tilde{x}_{2}\right)=-1+\sqrt{1-\left(\tilde{x}_{2}\right)^{2}}$.

First it is easy to verify that the constructed solution satisfies the Stokes equations with the incompressibility condition. The local Cartesian coordinate transformation matrix is

$$
A=\left(\begin{array}{cc}
\cos \theta^{*} & -\sin \theta^{*} \\
\sin \theta^{*} & \cos \theta^{*}
\end{array}\right)
$$

where $\theta^{*}$ is the angle between the normal vector and the $x_{1}$ axis at a point $\boldsymbol{x}^{*}$ on the interface.

From the interface force balance condition (1.7), we get the forces density

$$
f_{1}=\frac{3}{4} \cos ^{4} \theta \sin \theta-\frac{5}{4} \sin \theta \cos ^{2} \theta-\frac{1}{2} \sin ^{3} \theta
$$

and

$$
f_{2}=\frac{3}{4} \cos ^{3} \theta \sin ^{2} \theta+\frac{1}{8} \sin ^{2} \theta \cos \theta+\frac{1}{8} \cos ^{3} \theta .
$$


Here $\theta$ is the angle parameter. At the fixed interface point $\boldsymbol{x}^{*}$, it can be verified that

$$
\tilde{f}_{1}=-\frac{3}{8} \cos \theta^{*} \sin \theta^{*}
$$

and

$$
\tilde{f}_{2}=\frac{1}{2}+\frac{3}{8} \cos ^{2} \theta^{*}-\frac{3}{4} \cos ^{4} \theta^{*} .
$$

To verify Theorem 1.1, we derive the jump in pressure from the expression of $p$ to get

$$
[p]\left(\boldsymbol{x}^{*}\right)=\frac{3}{4} \cos ^{3} \theta^{*} \sin \theta^{*}-\frac{3}{8} \cos \theta^{*} \sin \theta^{*} .
$$

Using the local parameterization of the interface $\chi\left(\tilde{x}_{2}\right)$ evaluated at the local origin $\tilde{x}^{2}=0$, we get

$$
2\left[\mu \frac{\partial \boldsymbol{u}}{\partial n} \cdot \boldsymbol{n}\right]=\frac{3}{4} \cos ^{3} \theta^{*} \sin \theta^{*}
$$

So $[p]$ in Theorem 1.1 is verified for the example.

Similarly, we know that

$$
\left[\frac{\partial p}{\partial n}\right]\left(\boldsymbol{x}^{*}\right)=-\frac{3}{4} \sin \theta^{*} \cos \theta^{*}+3 \cos ^{3} \theta^{*} \sin \theta^{*} .
$$

To verify (3.15), we evaluate each term from the given expressions of $u_{i}, g_{i}$ and $p$ to get

$$
[\boldsymbol{G} \cdot \boldsymbol{n}]=-\frac{3}{2} \sin \theta^{*} \cos \theta^{*}+3 \cos ^{3} \theta^{*} \sin \theta^{*} .
$$

Using the relation

$$
\tilde{x}_{2}=\sin \left(\theta-\theta^{*}\right),
$$

we also get

$$
\frac{d(\boldsymbol{f} \cdot \boldsymbol{\tau})}{d \tilde{x}_{2}}\left(\boldsymbol{x}^{*}\right)=3 \cos ^{3} \theta^{*} \sin \theta^{*}-\frac{3}{4} \cos \theta^{*} \sin \theta^{*}
$$

and

$$
\begin{aligned}
& 2\left[\mu \frac{\partial^{2} \boldsymbol{u}}{\partial \tilde{x}^{2} \partial \tilde{x}^{2}}\right]\left(\boldsymbol{x}^{*}\right) \cdot \boldsymbol{n}\left(\boldsymbol{x}^{*}\right)=\frac{3}{2} \sin \theta^{*} \cos \theta^{*}-\frac{3}{2} \sin \theta^{*} \cos ^{3} \theta^{*} \\
& -4 \kappa\left[\mu \frac{\partial \boldsymbol{u}}{\partial \tilde{x}_{2}}\right]\left(\boldsymbol{x}^{*}\right) \cdot \boldsymbol{\tau}\left(\boldsymbol{x}^{*}\right)=-\frac{3}{2} \cos ^{3} \theta^{*} \sin \theta^{*}
\end{aligned}
$$

So $\left[\frac{\partial p}{\partial n}\right]$ in $(3.15)$ is verified for the example. 
4. The derivation of the jump condition for $\frac{\partial p}{\partial n}$ in $3 D$. The jump condition for $\frac{\partial p}{\partial n}$ in three dimensions derived in this section is new. The use of tensor notations and the local coordinate system make the result and the derivation parallel to those in two dimensions. In three dimensions, the interface $\Gamma$ is usually a surface and the tangential directions are not unique. At a fixed point on the interface, we can select two orthonormal tangential directions in addition to the normal direction to form a local coordinate system. In [1], a numerical method is proposed to determine the local coordinate system based on the level set representation of the interface. The transformation matrix at a fixed point $\boldsymbol{X}^{*}$ on the interface can be written as

$$
A=\left(\begin{array}{ccc}
n_{1}\left(\boldsymbol{X}^{*}\right) & \tau_{1}\left(\boldsymbol{X}^{*}\right) & \eta_{1}\left(\boldsymbol{X}^{*}\right) \\
n_{2}\left(\boldsymbol{X}^{*}\right) & \tau_{2}\left(\boldsymbol{X}^{*}\right) & \eta_{2}\left(\boldsymbol{X}^{*}\right) \\
n_{3}\left(\boldsymbol{X}^{*}\right) & \tau_{3}\left(\boldsymbol{X}^{*}\right) & \eta_{3}\left(\boldsymbol{X}^{*}\right)
\end{array}\right)
$$

where $\tau_{i}$ and $\eta_{i}$ are two unit orthonormal tangential vectors. In a neighborhood of the origin, the interface can be written as

$$
\Gamma=\left\{\left(\tilde{x}_{1}, \tilde{x}_{2}, \tilde{x}_{3}\right)=\left(\chi\left(\tilde{x}_{2}, \tilde{x}_{3}\right), \tilde{x}_{2}, \tilde{x}_{3}\right)\right\},
$$

where $\chi(0,0)=0, \frac{\partial \chi}{\partial \tilde{x}_{2}}(0,0)=0$ and $\frac{\partial \chi}{\partial \tilde{x}_{3}}(0,0)=0$.

We can define a level set function as $\varphi\left(\tilde{x}_{1}, \tilde{x}_{2}, \tilde{x}_{3}\right)=\tilde{x}_{1}-\chi\left(\tilde{x}_{2}, \tilde{x}_{3}\right)$ whose zero level set represents the interface $\Gamma$. Then the normal direction in a neighborhood of the origin in the local coordinate system is $\tilde{\boldsymbol{n}}=\nabla \varphi /|\nabla \varphi|$. The two unit tangential directions can be chosen as [12]

$$
\begin{gathered}
\tilde{\boldsymbol{\tau}}=\frac{1}{\sqrt{1+\left(\frac{\partial \chi}{\partial \tilde{x}_{2}}\right)^{2}}}\left(\frac{\partial \chi}{\partial \tilde{x}_{2}}, 1,0\right), \\
\tilde{\boldsymbol{\eta}}=\frac{1}{\sqrt{1+\left(\frac{\partial \chi}{\partial \tilde{x}_{3}}\right)^{2}}}\left(\frac{\partial \chi}{\partial \tilde{x}_{3}}, 0,1\right),
\end{gathered}
$$

respectively. The local unit normal direction is

$$
\tilde{\boldsymbol{n}}=\frac{\nabla \varphi}{\|\nabla \varphi\|}=\frac{\tilde{\boldsymbol{\tau}} \times \tilde{\boldsymbol{\eta}}}{\|\tilde{\boldsymbol{\tau}} \times \tilde{\boldsymbol{\eta}}\|}=\frac{1}{\sqrt{1+\left(\frac{\partial \chi}{\partial \tilde{x}_{2}}\right)^{2}+\left(\frac{\partial \chi}{\partial \tilde{x}_{3}}\right)^{2}}}\left(1,-\frac{\partial \chi}{\partial \tilde{x}_{2}},-\frac{\partial \chi}{\partial \tilde{x}_{3}}\right) .
$$

An obvious jump condition for $\frac{\partial p}{\partial n}$ in $3 \mathrm{D}$ is

$$
\left[\frac{\partial p}{\partial n}\right]=\left[\mu\left(\frac{\partial^{2} \tilde{u}_{1}}{\partial \tilde{x}_{1} \partial \tilde{x}_{1}}+\frac{\partial^{2} \tilde{u}_{1}}{\partial \tilde{x}_{2} \partial \tilde{x}_{2}}+\frac{\partial^{2} \tilde{u}_{1}}{\partial \tilde{x}_{3} \partial \tilde{x}_{3}}\right)\right]+\left[G_{i} n_{i}\right]=[\mu \Delta \boldsymbol{u} \cdot \boldsymbol{n}]+[\boldsymbol{G} \cdot \boldsymbol{n}]
$$

which contains the jump conditions of second order partial derivatives of the velocity.

Our main new result in three dimensions is the following theorem.

Theorem 4.1. Let $\boldsymbol{X}^{*}$ be a point on the interface $\Gamma \in C^{2}$. Let $\left\{\tilde{x}_{j}\right\}=$ $\left(\boldsymbol{n}\left(\boldsymbol{X}^{*}\right), \boldsymbol{\tau}\left(\boldsymbol{X}^{*}\right), \boldsymbol{\eta}\left(\boldsymbol{X}^{*}\right)\right), j=1,2,3$, be the local coordinate system determined by 
(2.1) and (4.1). Then the following is true

$$
\begin{aligned}
{\left[\frac{\partial p}{\partial \tilde{n}}\right]=} & {[\tilde{\boldsymbol{G}} \cdot \tilde{\boldsymbol{n}}]+\frac{\partial}{\partial \tilde{x}_{2}} \hat{f}_{2}+\frac{\partial}{\partial \tilde{x}_{3}} \hat{f}_{3}+2\left(\left[\mu \frac{\partial^{2} \tilde{u}_{1}}{\partial \tilde{x}_{2} \partial \tilde{x}_{2}}\right]+\left[\mu \frac{\partial^{2} \tilde{u}_{1}}{\partial \tilde{x}_{3} \partial \tilde{x}_{3}}\right]\right) } \\
& -4\left(\left[\mu \frac{\partial \tilde{u}_{2}}{\partial \tilde{x}_{2}}\right] \frac{\partial^{2} \chi}{\partial \tilde{x}_{2} \partial \tilde{x}_{2}}+\left[\mu \frac{\partial \tilde{u}_{3}}{\partial \tilde{x}_{3}}\right] \frac{\partial^{2} \chi}{\partial \tilde{x}_{3} \partial \tilde{x}_{3}}\right) \\
& -2\left\{\left[\mu \frac{\partial \tilde{u}_{2}}{\partial \tilde{x}_{2}}\right] \frac{\partial^{2} \chi}{\partial \tilde{x}_{3} \partial \tilde{x}_{3}}+\left[\mu \frac{\partial \tilde{u}_{3}}{\partial \tilde{x}_{3}}\right] \frac{\partial^{2} \chi}{\partial \tilde{x}_{2} \partial \tilde{x}_{2}}\right. \\
+ & {\left.\left[\mu \frac{\partial \tilde{u}_{3}}{\partial \tilde{x}_{2}}\right] \frac{\partial^{2} \chi}{\partial \tilde{x}_{2} \partial \tilde{x}_{3}}+\left[\mu \frac{\partial \tilde{u}_{2}}{\partial \tilde{x}_{3}}\right] \frac{\partial^{2} \chi}{\partial \tilde{x}_{2} \partial \tilde{x}_{3}}\right\} }
\end{aligned}
$$

at the origin $\left(\tilde{x}_{j}=0\right)$ in the local coordinate system, and $\tilde{u}_{1}=\boldsymbol{u} \cdot \boldsymbol{n}\left(\boldsymbol{X}^{*}\right), \tilde{u}_{2}=$ $\boldsymbol{u} \cdot \boldsymbol{\tau}\left(\boldsymbol{X}^{*}\right)$, and $\tilde{u}_{3}=\boldsymbol{u} \cdot \boldsymbol{\eta}\left(\boldsymbol{X}^{*}\right)$ are the velocity component in the normal and tangential directions. Note that all the quantities in the equality above are evaluated at the origin in the local coordinate system.

Proof. We re-write the expressions (1.8) and (1.9) in $\tilde{x}_{i}$ coordinate system to get

$$
\begin{aligned}
& {\left[\mu\left(\frac{\partial \tilde{u}_{i}}{\partial \tilde{n}} \tilde{\tau}_{i}+\frac{\partial \tilde{u}_{j}}{\partial \tilde{\tau}} \tilde{n}_{j}\right)\right]+\tilde{f}_{i} \tilde{\tau}_{i}=0,} \\
& {\left[\mu\left(\frac{\partial \tilde{u}_{i}}{\partial \tilde{n}} \tilde{\eta}_{i}+\frac{\partial \tilde{u}_{j}}{\partial \tilde{\eta}} \tilde{n}_{j}\right)\right]+\tilde{f}_{i} \tilde{\eta}_{i}=0 .}
\end{aligned}
$$

Similar to the proof in two dimensions, the crucial step is to simplify derivatives of the expressions above at the origin of the local coordinate system, and combine with (4.6) to get the desired result.

We differentiate Eq. (4.8) with respect to $\tilde{x}_{2}$ to get

$$
\left[\mu \frac{\partial}{\partial \tilde{x}_{2}}\left(\frac{\partial \tilde{u}_{i}}{\partial \tilde{n}} \tilde{\tau}_{i}+\frac{\partial \tilde{u}_{j}}{\partial \tilde{\tau}} \tilde{n}_{j}\right)\right](0,0)+\frac{\partial\left(\tilde{f}_{i} \tilde{\tau}_{i}\right)}{\partial \tilde{x}_{2}}(0,0)=0
$$

or

$$
\left[\mu\left(\frac{\partial^{2} \tilde{u}_{i}}{\partial \tilde{x}_{2} \partial \tilde{n}} \tilde{\tau}_{i}+\frac{\partial \tilde{u}_{i}}{\partial \tilde{n}} \frac{\partial \tilde{\tau}_{i}}{\partial \tilde{x}_{2}}+\frac{\partial^{2} \tilde{u}_{j}}{\partial \tilde{x}_{2} \partial \tilde{\tau}} \tilde{n}_{j}+\frac{\partial \tilde{u}_{j}}{\partial \tilde{\tau}} \frac{\partial \tilde{n}_{j}}{\partial \tilde{x}_{2}}\right)\right](0,0)+\frac{\partial\left(\tilde{f}_{i} \tilde{\tau}_{i}\right)}{\partial \tilde{x}_{2}}(0,0)=0
$$

We use the explicit expression of the interface in the local coordinate system to 
simplify terms. For example, for $\frac{\partial^{2} \tilde{u}_{i}}{\partial \tilde{x}_{2} \partial \tilde{n}}$ term, we have

$$
\begin{gathered}
\frac{\partial^{2} \tilde{u}_{i}}{\partial \tilde{x}_{2} \partial \tilde{n}} \\
=\frac{1}{\sqrt{1+\left(\frac{\partial \chi}{\partial \tilde{x}_{2}}\right)^{2}}} \frac{1}{\sqrt{1+\left(\frac{\partial \chi}{\partial \tilde{x}_{2}}\right)^{2}+\left(\frac{\partial \chi}{\partial \tilde{x}_{3}}\right)^{2}}}\left(\frac{\partial^{2} \tilde{u}_{i}}{\partial \tilde{x}_{1} \partial \tilde{x}_{1}}-\frac{\partial^{2} \tilde{u}_{i}}{\partial \tilde{x}_{1} \partial \tilde{x}_{2}} \frac{\partial \chi}{\partial \tilde{x}_{2}}-\frac{\partial^{2} \tilde{u}_{i}}{\partial \tilde{x}_{1} \partial \tilde{x}_{3}} \frac{\partial \chi}{\partial \tilde{x}_{3}}\right) \frac{\partial \chi}{\partial \tilde{x}_{2}} \\
-\frac{1}{\sqrt{1+\left(\frac{\partial \chi}{\partial \tilde{x}_{2}}\right)^{2}}} \frac{\frac{\partial \chi}{\partial \tilde{x}_{2}} \frac{\partial^{2} \chi}{\partial \tilde{x}_{2} \partial \tilde{x}_{2}}+\frac{\partial \chi}{\partial \tilde{x}_{3}} \frac{\partial^{2} \chi}{\partial \tilde{x}_{2} \partial \tilde{x}_{3}}}{\left(1+\left(\frac{\partial \chi}{\partial \tilde{x}_{2}}\right)^{2}+\left(\frac{\partial \chi}{\partial \tilde{x}^{3}}\right)^{2}\right)^{3 / 2}}\left(\frac{\partial \tilde{u}_{i}}{\partial \tilde{x}_{1}}-\frac{\partial \tilde{u}_{i}}{\partial \tilde{x}_{2}} \frac{\partial \chi}{\partial \tilde{x}_{2}}-\frac{\partial \tilde{u}_{i}}{\partial \tilde{x}_{3}} \frac{\partial \chi}{\partial \tilde{x}_{3}}\right) \\
+\frac{1}{\sqrt{1+\left(\frac{\partial \chi}{\partial \tilde{x}_{2}}\right)^{2}}} \frac{\partial^{2} \tilde{u}_{i}}{\sqrt{1+\left(\frac{\partial \chi}{\partial \tilde{x}_{2}}\right)^{2}+\left(\frac{\partial \chi}{\partial \tilde{x}_{3}}\right)^{2}}}\left(\frac{\partial \tilde{u}_{i}}{\partial \tilde{x}_{2}} \frac{\partial^{2} \chi}{\partial \tilde{x}_{2} \partial \tilde{x}_{2}}-\frac{\partial^{2} \tilde{u}_{i}}{\partial \tilde{x}_{2} \partial \tilde{x}_{2}} \frac{\partial \chi}{\partial \tilde{x}_{2}}\right. \\
\left.\frac{\tilde{x}_{2} \partial \tilde{x}_{3}}{\partial \tilde{x}_{3}}-\frac{\partial \chi \tilde{u}_{i}}{\partial \tilde{x}_{3}} \frac{\partial^{2} \chi}{\partial \tilde{x}_{2} \partial \tilde{x}_{3}}\right) .
\end{gathered}
$$

After some manipulations and arranging terms [12], we simplify the expression (4.11) to

$$
\begin{aligned}
{\left[\mu \left(\frac{\partial^{2} \tilde{u}_{2}}{\partial \tilde{x}_{1} \partial \tilde{x}_{2}}-2 \frac{\partial \tilde{u}_{2}}{\partial \tilde{x}_{2}} \frac{\partial^{2} \chi}{\partial \tilde{x}_{2} \partial \tilde{x}_{2}}-\frac{\partial \tilde{u}_{2}}{\partial \tilde{x}_{3}} \frac{\partial^{2} \chi}{\partial \tilde{x}_{2} \partial \tilde{x}_{3}}+2 \frac{\partial \tilde{u}_{1}}{\partial \tilde{x}_{1}} \frac{\partial^{2} \chi}{\partial \tilde{x}_{2} \partial \tilde{x}_{2}}\right.\right.} \\
\left.\left.+\frac{\partial^{2} \tilde{u}_{1}}{\partial \tilde{x}_{2} \partial \tilde{x}_{2}}-\frac{\partial \tilde{u}_{3}}{\partial \tilde{x}_{2}} \frac{\partial^{2} \chi}{\partial \tilde{x}_{2} \partial \tilde{x}_{3}}\right)\right](0,0)+\frac{\partial\left(\tilde{f}_{i} \tilde{\tau}_{i}\right)}{\partial \tilde{x}_{2}}(0,0)=0 .
\end{aligned}
$$

Similarly, we differentiate the expression (4.9) with respect to $\tilde{x}_{3}$ to get

$$
\left[\mu \frac{\partial}{\partial \tilde{x}_{3}}\left(\frac{\partial \tilde{u}_{i}}{\partial \tilde{n}} \tilde{\eta}_{i}+\frac{\partial \tilde{u}_{j}}{\partial \tilde{\eta}} \tilde{n}_{j}\right)\right](0,0)+\frac{\partial\left(\tilde{f}_{i} \tilde{\eta}_{i}\right)}{\partial \tilde{x}_{3}}(0,0)=0
$$

or

$$
\left[\mu\left(\frac{\partial^{2} \tilde{u}_{i}}{\partial \tilde{x}_{3} \partial \tilde{n}} \tilde{\eta}_{i}+\frac{\partial \tilde{u}_{i}}{\partial \tilde{n}} \frac{\partial \tilde{\eta}_{i}}{\partial \tilde{x}_{3}}+\frac{\partial^{2} \tilde{u}_{j}}{\partial \tilde{x}_{3} \partial \tilde{\eta}} \tilde{n}_{j}+\frac{\partial \tilde{u}_{j}}{\partial \tilde{\eta}} \frac{\partial \tilde{n}_{j}}{\partial \tilde{x}_{3}}\right)\right](0,0)+\frac{\partial\left(\tilde{f}_{i} \tilde{\eta}_{i}\right)}{\partial \tilde{x}_{3}}(0,0)=0 .
$$

Again, we take one term in the expression above as an example of how to express it 
in terms of the interface quantities and the velocity. For $\frac{\partial^{2} \tilde{u}_{i}}{\partial \tilde{x}_{3} \partial \tilde{n}}$ term, we have

$$
\begin{aligned}
& \frac{\partial^{2} \tilde{u}_{i}}{\partial \tilde{x}_{3} \partial \tilde{n}} \\
& =\frac{1}{\sqrt{1+\left(\frac{\partial \chi}{\partial \tilde{x}_{3}}\right)^{2}}} \frac{1}{\sqrt{1+\left(\frac{\partial \chi}{\partial \tilde{x}_{2}}\right)^{2}+\left(\frac{\partial \chi}{\partial \tilde{x}_{3}}\right)^{2}}}\left(\frac{\partial^{2} \tilde{u}_{i}}{\partial \tilde{x}_{1} \partial \tilde{x}_{1}}-\frac{\partial^{2} \tilde{u}_{i}}{\partial \tilde{x}_{1} \partial \tilde{x}_{2}} \frac{\partial \chi}{\partial \tilde{x}_{2}}-\frac{\partial^{2} \tilde{u}_{i}}{\partial \tilde{x}_{1} \partial \tilde{x}_{3}} \frac{\partial \chi}{\partial \tilde{x}_{3}}\right) \frac{\partial \chi}{\partial \tilde{x}_{3}} \\
& -\frac{1}{\sqrt{1+\left(\frac{\partial \chi}{\partial \tilde{x}_{3}}\right)^{2}}} \frac{\frac{\partial \chi}{\partial \tilde{x}_{2}} \frac{\partial^{2} \chi}{\partial \tilde{x}_{2} \partial \tilde{x}_{3}}+\frac{\partial \chi}{\partial \tilde{x}_{3}} \frac{\partial^{2} \chi}{\partial \tilde{x}_{3} \partial \tilde{x}_{3}}}{\left(1+\left(\frac{\partial \chi}{\partial \tilde{x}_{2}}\right)^{2}+\left(\frac{\partial \chi}{\partial \tilde{x}_{3}}\right)^{2}\right)^{3 / 2}}\left(\frac{\partial \tilde{u}_{i}}{\partial \tilde{x}_{1}}-\frac{\partial \tilde{u}_{i}}{\partial \tilde{x}_{2}} \frac{\partial \chi}{\partial \tilde{x}_{2}}-\frac{\partial \tilde{u}_{i}}{\partial \tilde{x}_{3}} \frac{\partial \chi}{\partial \tilde{x}_{3}}\right) \\
& +\frac{1}{\sqrt{1+\left(\frac{\partial \chi}{\partial \tilde{x}_{3}}\right)^{2}}} \frac{1}{\sqrt{1+\left(\frac{\partial \chi}{\partial \tilde{x}_{2}}\right)^{2}+\left(\frac{\partial \chi}{\partial \tilde{x}_{3}}\right)^{2}}}\left(\frac{\partial^{2} \tilde{u}_{i}}{\partial \tilde{x}_{1} \partial \tilde{x}_{3}}-\frac{\partial^{2} \tilde{u}_{i}}{\partial \tilde{x}_{2} \partial \tilde{x}_{3}} \frac{\partial \chi}{\partial \tilde{x}_{2}}\right. \\
& \left.-\frac{\partial \tilde{u}_{i}}{\partial \tilde{x}_{2}} \frac{\partial^{2} \chi}{\partial \tilde{x}_{2} \partial \tilde{x}_{3}}-\frac{\partial^{2} \tilde{u}_{i}}{\partial \tilde{x}_{3} \partial \tilde{x}_{3}} \frac{\partial \chi}{\partial \tilde{x}_{3}}-\frac{\partial \tilde{u}_{i}}{\partial \tilde{x}_{3}} \frac{\partial^{2} \chi}{\partial \tilde{x}_{3} \partial \tilde{x}_{3}}\right) .
\end{aligned}
$$

After some manipulations and arranging terms, we can simplify the expression (4.14) to

$$
\begin{gathered}
{\left[\mu \left(\frac{\partial^{2} \tilde{u}_{3}}{\partial \tilde{x}_{1} \partial \tilde{x}_{3}}-2 \frac{\partial \tilde{u}_{3}}{\partial \tilde{x}_{3}} \frac{\partial^{2} \chi}{\partial \tilde{x}_{3} \partial \tilde{x}_{3}}-\frac{\partial \tilde{u}_{3}}{\partial \tilde{x}_{2}} \frac{\partial^{2} \chi}{\partial \tilde{x}_{2} \partial \tilde{x}_{3}}+2 \frac{\partial \tilde{u}_{1}}{\partial \tilde{x}_{1}} \frac{\partial^{2} \chi}{\partial \tilde{x}_{3} \partial \tilde{x}_{3}}\right.\right.} \\
\left.\left.+\frac{\partial^{2} \tilde{u}_{1}}{\partial \tilde{x}_{3} \partial \tilde{x}_{3}}-\frac{\partial \tilde{u}_{2}}{\partial \tilde{x}_{3}} \frac{\partial^{2} \chi}{\partial \tilde{x}_{2} \partial \tilde{x}_{3}}\right)\right](0,0)+\frac{\partial\left(\tilde{f}_{i} \tilde{\eta}_{i}\right)}{\partial \tilde{x}_{3}}(0,0)=0 .
\end{gathered}
$$

We can observe the expected symmetry between expressions (4.12) and (4.15).

We add expressions (4.6), (4.11), and (4.14) together to get:

$$
\begin{aligned}
& {\left[\frac{\partial p}{\partial n}\right](0,0) } \\
= & {\left[\mu\left(\frac{\partial^{2} \tilde{u}_{1}}{\partial \tilde{x}_{1} \partial \tilde{x}_{1}}+\frac{\partial^{2} \tilde{u}_{1}}{\partial \tilde{x}_{2} \partial \tilde{x}_{2}}+\frac{\partial^{2} \tilde{u}_{1}}{\partial \tilde{x}_{3} \partial \tilde{x}_{3}}\right)\right](0,0)+\left[\tilde{G}_{i} \tilde{n}_{i}\right](0,0) } \\
+ & {\left[\mu\left(\frac{\partial^{2} \tilde{u}_{i}}{\partial \tilde{x}_{2} \partial \tilde{n}} \tilde{\tau}_{i}+\frac{\partial \tilde{u}_{i}}{\partial \tilde{n}} \frac{\partial \tilde{\tau}_{i}}{\partial \tilde{x}_{2}}+\frac{\partial^{2} \tilde{u}_{j}}{\partial \tilde{x}_{2} \partial \tilde{\tau}} \tilde{n}_{j}+\frac{\partial \tilde{u}_{j}}{\partial \tilde{\tau}} \frac{\partial \tilde{n}_{j}}{\partial \tilde{x}_{2}}\right)\right](0,0)+\frac{\partial\left(\tilde{f}_{i} \tilde{\tau}_{i}\right)}{\partial \tilde{x}_{2}}(0,0) } \\
& +\left[\mu\left(\frac{\partial^{2} \tilde{u}_{i}}{\partial \tilde{x}_{3} \partial \tilde{n}} \tilde{\eta}_{i}+\frac{\partial \tilde{u}_{i}}{\partial \tilde{n}} \frac{\partial \tilde{\eta}_{i}}{\partial \tilde{x}_{3}}+\frac{\partial^{2} \tilde{u}_{j}}{\partial \tilde{x}_{3} \partial \tilde{\eta}} \tilde{n}_{j}+\frac{\partial \tilde{u}_{j}}{\partial \tilde{\eta}} \frac{\partial \tilde{n}_{j}}{\partial \tilde{x}_{3}}\right)\right](0,0)+\frac{\partial\left(\tilde{f}_{i} \tilde{\eta}_{i}\right)}{\partial \tilde{x}_{3}}(0,0) .
\end{aligned}
$$

From the incompressibility condition we have (after we differentiate with respect to $\left.\tilde{x}_{1}\right)$

$$
\frac{\partial^{2} \tilde{u}_{1}}{\partial \tilde{x}_{1} \partial \tilde{x}_{1}}+\frac{\partial^{2} \tilde{u}_{2}}{\partial \tilde{x}_{1} \partial \tilde{x}_{2}}+\frac{\partial^{2} \tilde{u}_{3}}{\partial \tilde{x}_{1} \partial \tilde{x}_{3}}=0 .
$$

Finally, we plug in the expressions (4.12), (4.15), (4.17), and other similar terms 
into the expression (4.16) to get [12]

$$
\begin{aligned}
& {\left[\frac{\partial p}{\partial n}\right](0,0) } \\
= & {\left[\mu\left(-2 \frac{\partial \tilde{u}_{2}}{\partial \tilde{x}_{2}} \frac{\partial^{2} \chi}{\partial \tilde{x}_{2} \partial \tilde{x}_{2}}-2 \frac{\partial \tilde{u}_{2}}{\partial \tilde{x}_{3}} \frac{\partial^{2} \chi}{\partial \tilde{x}_{2} \partial \tilde{x}_{3}}+2 \frac{\partial \tilde{u}_{1}}{\partial \tilde{x}_{1}} \frac{\partial^{2} \chi}{\partial \tilde{x}_{2} \partial \tilde{x}_{2}}+2 \frac{\partial^{2} \tilde{u}_{1}}{\partial \tilde{x}_{2} \partial \tilde{x}_{2}}\right)\right](0,0) } \\
& +\left[\mu\left(-2 \frac{\partial \tilde{u}_{3}}{\partial \tilde{x}_{2}} \frac{\partial^{2} \chi}{\partial \tilde{x}_{2} \partial \tilde{x}_{3}}-2 \frac{\partial \tilde{u}_{3}}{\partial \tilde{x}_{3}} \frac{\partial^{2} \chi}{\partial \tilde{x}_{3} \partial \tilde{x}_{3}}+2 \frac{\partial \tilde{u}_{1}}{\partial \tilde{x}_{1}} \frac{\partial^{2} \chi}{\partial \tilde{x}_{3} \partial \tilde{x}_{3}}+2 \frac{\partial^{2} \tilde{u}_{1}}{\partial \tilde{x}_{3} \partial \tilde{x}_{3}}\right)\right](0,0) \\
& +\frac{\partial\left(\tilde{f}_{i} \tilde{\tau}_{i}\right)}{\partial \tilde{x}_{2}}(0,0)+\frac{\partial\left(\tilde{f}_{i} \tilde{\eta}_{i}\right)}{\partial \tilde{x}_{3}}(0,0)+\left[\tilde{G}_{i} \tilde{n}_{i}\right](0,0) \\
= & {\left.\left[-4 \frac{\partial \tilde{u}_{2}}{\partial \tilde{x}_{2}} \frac{\partial^{2} \chi}{\partial \tilde{x}_{2} \partial \tilde{x}_{2}}-2 \frac{\partial \tilde{u}_{2}}{\partial \tilde{x}_{3}} \frac{\partial^{2} \chi}{\partial \tilde{x}_{2} \partial \tilde{x}_{3}}-2 \frac{\partial \tilde{u}_{3}}{\partial \tilde{x}_{3}} \frac{\partial^{2} \chi}{\partial \tilde{x}_{2} \partial \tilde{x}_{2}}+2 \frac{\partial^{2} \tilde{u}_{1}}{\partial \tilde{x}_{2} \partial \tilde{x}_{2}}\right)\right](0,0) } \\
& +\left[\mu\left(-2 \frac{\partial \tilde{u}_{3}}{\partial \tilde{x}_{2}} \frac{\partial^{2} \chi}{\partial \tilde{x}_{2} \partial \tilde{x}_{3}}-4 \frac{\partial \tilde{u}_{3}}{\partial \tilde{x}_{3}} \frac{\partial^{2} \chi}{\partial \tilde{x}_{3} \partial \tilde{x}_{3}}-2 \frac{\partial \tilde{u}_{2}}{\partial \tilde{x}_{2}} \frac{\partial^{2} \chi}{\partial \tilde{x}_{3} \partial \tilde{x}_{3}}+2 \frac{\partial^{2} \tilde{u}_{1}}{\partial \tilde{x}_{3} \partial \tilde{x}_{3}}\right)\right](0,0) \\
& +\frac{\partial\left(\tilde{f}_{i} \tilde{\tau}_{i}\right)}{\partial \tilde{x}_{2}}(0,0)+\frac{\partial\left(\tilde{f}_{i} \tilde{\eta}_{i}\right)}{\partial \tilde{x}_{3}}(0,0)+\left[\tilde{G}_{i} \tilde{n}_{i}\right](0,0) \cdot
\end{aligned}
$$

This completes the proof.

REMARK 4.2. The jump condition of the normal derivative of the pressure can be written as follows

$$
\begin{aligned}
{\left[\frac{\partial p}{\partial n}\right] } & {[\boldsymbol{G} \cdot \boldsymbol{n}]+\frac{\partial}{\partial \tilde{x}_{2}}(\boldsymbol{f} \cdot \boldsymbol{\tau})+\frac{\partial}{\partial \tilde{x}_{3}}(\boldsymbol{f} \cdot \boldsymbol{\eta})+2\left(\left[\mu \frac{\partial^{2} \boldsymbol{u}}{\partial \tilde{x}_{2} \partial \tilde{x}_{2}}\right] \cdot \boldsymbol{n}+\left[\mu \frac{\partial^{2} \boldsymbol{u}}{\partial \tilde{x}_{3} \partial \tilde{x}_{3}}\right] \cdot \boldsymbol{n}\right) } \\
= & 4\left(\left[\mu \frac{\partial \boldsymbol{u}}{\partial \tilde{x}_{2}}\right] \cdot \boldsymbol{\tau} \frac{\partial^{2} \chi}{\partial \tilde{x}_{2} \partial \tilde{x}_{2}}+\left[\mu \frac{\partial \boldsymbol{u}}{\partial \tilde{x}_{3}}\right] \cdot \boldsymbol{\eta} \frac{\partial^{2} \chi}{\partial \tilde{x}_{3} \partial \tilde{x}_{3}}\right) \\
-2 & \left(\left(\left[\mu \frac{\partial \boldsymbol{u}}{\partial \tilde{x}_{3}}\right] \cdot \boldsymbol{\tau}+\left[\mu \frac{\partial \boldsymbol{u}}{\partial \tilde{x}_{2}}\right] \cdot \boldsymbol{\eta}\right)\right) \frac{\partial^{2} \chi}{\partial \tilde{x}_{2} \partial \tilde{x}_{3}}+\left[\mu \frac{\partial \boldsymbol{u}}{\partial \tilde{x}_{3}}\right] \cdot \boldsymbol{\eta} \frac{\partial^{2} \chi}{\partial \tilde{x}_{2} \partial \tilde{x}_{2}} \\
& \left.+\left[\mu \frac{\partial \boldsymbol{u}}{\partial \tilde{x}_{2}}\right] \cdot \boldsymbol{\tau} \frac{\partial^{2} \chi}{\partial \tilde{x}_{3} \partial \tilde{x}_{3}}\right)
\end{aligned}
$$

with correct understanding of each term involved as discussed in the proof.

Acknowledgments. This work was partially supported by a USA NSF/NIH grant \#0201094, a USA ARO grant number 49308-MA, a US-AFSOR grant FA955006-1-0241, and a USA NSF grant DMS-0412654. We also would like to thank Dr. Sheng Xu of Cornell University for useful discussions.

\section{REFERENCES}

[1] S. Deng, K. Ito And Z. Li, Three dimensional elliptic solvers for interface problems and applications, J. Comput. Phys., 184 (2003), pp. 215-243. 
[2] K. Iто AND Z. LI, Interface relations for Stokes equations with discontinuous viscosity and singular sources, Applied Math. Letters,, 19 (2006), pp. 229-234.

[3] D. D. Joseph and Y. Y. Renardy, Fundamentals of two-fluid dynamics, Springer-Verlag, 1993.

[4] M. KAng, R. Fedkiw, AND X. LiU, A boundary condition capturing method for multiphase incompressible flow, J. Sci. Comput., 15 (2000), pp. 323-360.

[5] M-C. LAI ANd Z. LI, A remark on jump conditions for the three-dimensional Navier-Stokes equations involving an immersed moving membrane, Applied Math. Letters, 14 (2001), pp. $149-154$.

[6] R. J. LeVeque and Z. Li, Immersed interface method for Stokes flow with elastic boundaries or surface tension, SIAM J. Sci. Comput., 18 (1997), pp. 709-735.

[7] Z. LI, The Immersed Interface Method - A Numerical Approach for Partial Differential Equations with Interfaces, $\mathrm{PhD}$ thesis, University of Washington, 1994.

[8] Z. LI, An overview of the immersed interface method and its applications, Taiwanese J. Mathematics, 7 (2003), pp. 1-49.

[9] Z. Li And K. ITO, The Immersed Interface Method - Numerical Solutions of PDEs Involving Interfaces and Irregular Domains, SIAM Frontier Series in Applied Mathematics, FR33, 2006.

[10] S. R. Lubkin AND Z. LI, Force and deformation on branching rudiments: Cleaving between hypotheses, Biomechanics and Modeling in Mechanobiology, 1 (2002), pp. 5-16.

[11] J. Sokolowski and J. P. Zolesio, Introduction to Shape Optimization, Springer-Verlag, 1991.

[12] X. WAN, Numerical Simulation Methods for Biological Tissue Interactions, PhD thesis, North Carolina State University, 2006. 